\title{
ARTIGO
}

\section{ANÁLISE DE POLÍTICAS PÚBLICAS BRASILEIRAS EM CIÊNCIA, TECNOLOGIA E INOVAÇÃO COM FOCO NA CULTURA DE INOVAÇÃO E ATUAÇÃO INTEGRADA DE AGENTES DO SISTEMA DE INOVAÇÃO}

BRAZILIAN POLICIES IN SCIENCE, TECHNOLOGY AND INNOVATION FOCUSING INNOVATION CULTURE AND INTEGRATED ACTION OF AGENTS OF THE INNOVATION SYSTEM

${ }^{1}$ Elaine da Silva

Universidade Federal de São Carlos ${ }^{1}$

\section{Correspondência}

Elaine da Silva

Universidade Federal de São Carlos

São Carlos, SP - Brasil

E-mail: emaildoautor@email.com

(iD http://orcid.org/0000-0003-1449-354X

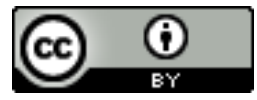

JITA: FJ. Knowledge management

e-Location: 019019 
RESUMO

Considerando a importância da inovação para os segmentos produtivo, acadêmico e governamental, se apresenta uma análise das políticas públicas de inovação vigentes no Brasil no que tange à promoção da inovação sistêmica e aberta, vislumbrando assim oportunidades e desafios que se apresentam a todos os envolvidos com a geração de inovação no país. Por meio da aplicação do método 'Análise de Conteúdo' as referidas políticas foram analisadas quanto à presença de cultura de inovação enfocando o conhecimento e ação integrada de agentes do sistema de inovação. Observou-se que as políticas públicas promovem a cooperação entre organizações que integram o sistema inovativo, entretanto não há evidências de incentivo ou diretrizes para a participação de pessoas não vinculadas a organizações, seguramente um desafio tanto para elaboradores de políticas quanto para todos que dedicam-se a promover a ampla participação de organizações e pessoas em atividades inovativas.

PALAVRAS-CHAVE:

Geração de conhecimento. Cultura de inovação. Sistemas de inovação. Inovação aberta. Políticas públicas de inovação.

\section{ABSTRACT}

Considering the importance of innovation for the productive, academic and governmental segments, this article presents an analysis of the public policies of innovation in Brazil with regards to the promotion of systemic and open innovation, envisioning opportunities and challenges that are presented to all those involved the generation of innovation in the country. Through the application of the 'Content Analysis' method, these policies were analyzed about the presence of culture of innovation focusing on the knowledge and integrated action of agents of the innovation system. It was observed that public policies promote cooperation between organizations that are part of the innovative system, but there is no evidence of incentive or guidelines for the participation of people not linked to organizations, certainly a challenge for both policymakers and all those who dedicate themselves, to promote the broad participation of organizations and individuals in innovative activities.

\section{KEYWORDS}

Knowledge generation. Innovation culture. Innovation systems. Open innovation. Innovation policies. 


\section{RDBCI

\section{Introdução}

Seguramente a sociedade e a atual situação das nações é resultado da acumulação de todas as descobertas, invenções, melhorias, aperfeiçoamentos e esforços de gerações que viveram antes de nós (LIST, 1841; FREEMAN; SOETE, 2008). Portanto, partindo do pressuposto de que invenções e melhorias significativas implementadas se constituem em inovações, é adequando afirmar que inovações sempre fizeram parte da trajetória da humanidade.

Descritas inicialmente como novas combinações em processos produtivos (SHUMPETER, 1982), as inovações se difundiram como elementos para vantagem competitiva e diferencial organizacional, concebidas e desenvolvidas no interior do contexto organizacional, num sistema fechado.

A partir da Década de 1980 emerge a abordagem sistêmica da inovação, que considera a inovação como resultado da atuação integrada de diferentes organizações com objetivos comuns; surgem então os Sistemas Nacionais de Inovação (SNI), caracterizados pela atuação integrada de agentes públicos e privados dos setores produtivo, de serviços, acadêmico e institucional. A inovação deixa de ser encarada como resultado de esforços das organizações isoladamente e passa a ser entendida como o produto da interação entre diferentes agentes. Esta concepção se revela bastante pertinente, posto que, em muitos países, é no âmbito de universidades e institutos de pesquisa que parte considerável do conhecimento, em especial conhecimento científico e tecnológico de fronteira, é produzida.

Mais recentemente, a partir da Década de 2010, com o advento das tecnologias de informação comunicação, principalmente das redes sociais, se observa o crescimento da participação da sociedade civil independente de vínculos organizacionais em processos inovativos, muitas vezes promovidos via internet em eventos conhecidos como 'Desafio de ideias'; ou 'Hackaton', caracterizados pelo incentivo a qualquer pessoa para contribuir com uma proposta, ideia ou solução para um processo inovativo idealizado por uma organização.

É importante que as nações estejam atentas aos meios de geração de inovação e que formulem políticas públicas capazes de promover a participação de todos os agentes e pessoas competentes a contribuir com geração de inovação e desenvolvimento do país. Nessa perspectiva, o objetivo do presente trabalho consiste em apresentar uma análise das políticas públicas de inovação vigentes no Brasil no que tange à promoção da inovação sistêmica e aberta, vislumbrando assim oportunidades e desafios que se apresentam a todos os envolvidos com a geração de inovação no país.

Para tanto se discute conceitos de inovação aberta, sistemas e ecossistemas de inovação.

\begin{tabular}{|c|c|}
\hline RDBCl. Rev Diait $B$ & Camninas SP \\
\hline
\end{tabular}


Se propõe uma reflexão acerca do papel de políticas públicas de inovação no contexto de SNI e são apresentadas as políticas públicas em CT\&I nacionais.

Na seções seguintes são esclarecidos os procedimentos metodológicos e a apresentação e análise de resultados, seções construídas a partir de um recorte da Tese de Doutorado de Silva (2018). Em sequência, são apresentadas as considerações finais.

\section{Inovação Aberta, Sistemas e Ecossistemas de Inovação}

A inovação aberta, conceito introduzido por Chesbrough (2003), entende que o ambiente externo à empresa oferece contribuições valiosas à atividade inovativa nas organizações.

De acordo com Organization for Economic Co-operation and Development (OECD), mudanças no ambiente de inovação, tais como a concorrência cada vez mais global e intensa, resultando em ciclos de vida mais curtos, e o conhecimento mais multidisciplinar, tornam a inovação mais cara e mais arriscada. Nesse contexto, surge a necessidade de empresas promoverem a geração de inovação em conjunto com clientes, fornecedores, concorrentes, universidades e institutos de pesquisa, etc. (ORGANISATION, 2008). Em complemento, Stal, Nohara e Chagas Júnior (2014, p.296), declaram que inovação aberta é o resultado da

\footnotetext{
[...] ação conjunta de várias fontes no processo de inovação, que utiliza ao mesmo tempo as competências internas da empresa, não só para realizar $\mathrm{P} \& \mathrm{D}$, como para procurar, selecionar e acessar oportunidades e ativos externos à empresa. Este modelo enxerga a inovação como o resultado da formação e atuação de redes de colaboração sistemáticas - não apenas pontuais - que oferecem conhecimento, ideias e patentes para a geração de novos produtos e processos.
}

O conceito que preconiza a atuação de diferentes agentes para o desenvolvimento de inovações se constitui no cerne da abordagem sistêmica da inovação, que "[...] enfatiza a importância da transferência e da difusão de ideias, experiências, conhecimentos, informações e sinais de vários tipos" (ORGANISATION..., 2005, p.41). Dessa maneira, se reconhece a influência de instituições externas ao contexto empresarial para a geração e implementação de inovação, que passa a ser entendida como um processo dinâmico, que tem por base a aprendizagem e a interação.

A abordagem sistêmica da inovação surge a partir da Década de 1980, quando uma nova configuração global consolida a perspectiva de reconhecer a inovação não mais como uma atividade isolada da empresa, mais sim fruto da geração de conhecimento e da interação entre vários agentes das esferas pública e privada. Lundvall et al. (2002, p.224, tradução nossa) afirmam que "[...] o novo contexto mais do que qualquer outra coisa é caracterizado por uma aceleração da taxa de mudança, dando uma maior importância aos processos de aprendizagem

\begin{tabular}{l|l|l|l|l|l|}
\hline C RDBCl: Rev. Digit. Bibliotecon. Cienc. Inf. & Campinas, SP & v.17 & $1-14$ & e019019 & 2019 \\
\hline
\end{tabular}


para o desempenho econômico". O conceito se popularizou rapidamente entre acadêmicos e formuladores de políticas públicas com enfoque no crescimento econômico e desenvolvimento.

De acordo com a ORGANISATION (1997, p.9, tradução nossa) um Sistema Nacional de Inovação, “[...] parte da premissa que a compreensão dos vínculos entre os agentes envolvidos em processos inovativos é fundamental para a melhoria do desempenho tecnológico".

Johnson (1992) declara ser um SNI composto pela inter-relação de todos os fatores institucionais e estruturais de um país que, geram, selecionam e difundem inovação. Edquist (1997) a seu turno, define SNI como todos os fatores econômicos, sociais, políticos, organizacionais e outros que influenciam o desenvolvimento, a difusão e o uso de inovações. Godin (2009) complementa afirmando que os elementos ou instituições componentes do SNI são empresas, laboratórios públicos, universidades, instituições financeiras, o sistema educacional de maneira geral, órgãos reguladores e governamentais atuando de modo integrado. Figueiredo (2012) contribui com a elucidação de quais instituições podem integrar um SNI quando afirma tratar-se de

[...] um conjunto de organizações que envolve empresas e várias organizações de suporte, tais como universidades, institutos de pesquisa, centros de formação e treinamento, escolas técnicas, empresas de consultoria, organizações de metrologia e patentárias etc. (FIGUEIREDO, 2012, p.6).

Com o advento da internet, e principalmente das redes sociais, as interações com foco na geração de inovação vão além das relações institucionais formais e passam a abrigar também contribuições de pessoas individualmente, tais como estudantes e ou profissionais autônomos, sem vínculos institucionais.

À medida em que processos inovativos passam a incorporar a participação da sociedade civil e relações dinâmicas entre os agentes, sem necessariamente uma relação contratual formalizada, se percebe a pertinência de promover ecossistemas de inovação, em que os recursos de pessoas, capital e know-how são fluidos, e o ritmo das transações é impulsionado por uma busca incessante de oportunidades e financiamentos.

De acordo com Moore (2006) um ecossistema de inovação compreende o estabelecimento de uma visão de futuro, que deve ser compartilhada pelos agentes envolvidos, que cooperam, competem e evoluem num ambiente criador de oportunidades. Destarte, um ecossistema de inovação consiste em uma rede de relações na qual fluem informações e talentos por meio da cocriação e de valor sustentado (ETZCOWITZ; LEYDESDORFF, 2000). 


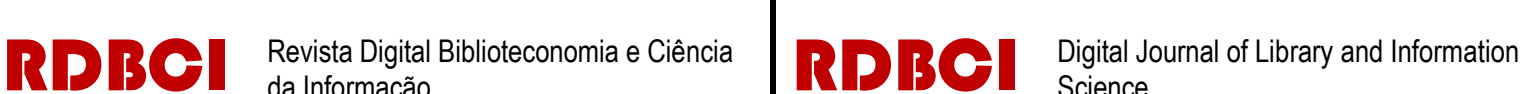

A partir da abordagem sistêmica da inovação e diante da importância de se tornarem nações mais inovadoras, países estabelecem políticas públicas com o intuito de direcionar, fomentar e/ou promover a atividade inovativa, que por consequência, espera-se que traga desenvolvimento às nações. Perspectiva em que se destaca o papel das políticas públicas de inovação

\footnotetext{
[...] que passam a ter ênfase na interação entre instituições e observam processos interativos na criação, difusão e aplicação de conhecimentos. Elas ressaltam a importância das condições, regulações e políticas em que os mercados operam e, por consequência, o papel dos governos em monitorar e buscar a harmonia nessa estrutura geral (ORGANISATION..., 2005, p.41).
}

Considerando a orientação para a atuação sistêmica, no que tange à geração de inovação, é seguro afirmar que "[...] o ambiente nacional pode ter uma considerável influência para estimular, facilitar retardar ou impedir as atividades inovativas das firmas" (FREEMAN; SOETE, 2008, p.503), dessa maneira, a análise de políticas públicas de inovação seguramente oferece um contributo importante para a detecção de oportunidades e desafios para o desenvolvimento da atividade inovativa de uma nação.

\section{Papel de Políticas Públicas de Inovação no Contexto de SNI}

Segundo Lundvall e Borrás (1997, p.13, tradução nossa) “[...] as políticas de inovação das nações devem ter como principal objetivo contribuir para a capacidade de aprendizagem das pessoas e das organizações". Porquanto as questões relacionadas à rapidez das transformações globais, a abordagem sistêmica de inovação e a centralidade da aprendizagem e do conhecimento devem integrar as preocupações dos elaboradores de políticas públicas de inovação. Todavia, há que se considerar a complexidade intrínseca ao estabelecimento de políticas públicas, ressaltada por Fernandes (2007, p.203), ao evidenciar que

\footnotetext{
Apesar de se tratar de uma área técnico-administrativa a esfera das políticas públicas também possui uma dimensão política uma vez que está relacionada ao processo decisório. Isto é, ao Estado é imperativo fazer escolhas sobre que área social atuar, onde atuar, por que atuar e quando atuar. Estas escolhas, por parte do Estado, que se transformam em decisões são condicionadas por interesses de diversos grupos sociais. Representam conquistas que se traduzem legalmente em direitos ou garantias defendidos pela sociedade.
}

De maneira genérica, políticas públicas de inovação são tradicionalmente concebidas como ferramentas para apoiar a produção e implementação de inovações de países ou regiões e seus respectivos SI. O debate acadêmico e político sobre políticas públicas de inovação tem se concentrado, principalmente, no apoio à geração de inovações. Tal fato é ratificado pelos conceitos e indicadores de inovação, que geralmente se dedicam a avaliar e comparar a 
competitividade entre países, enfocando, na maioria das vezes, questões relacionadas mormente à oferta de inovação (CORNELL UNIVERSITY; INSEAD; WIPO, 2016).

Considera-se importante, no âmbito da elaboração de políticas públicas de inovação, a análise acerca do enfoque na oferta (supply-side) e, também, na demanda (demand-side) por inovação. Nessa perspectiva, são elencadas três funções da inovação que potencialmente devem nortear a elaboração de políticas públicas de inovação, são elas:

- A primeira função da inovação é impulsionar o desenvolvimento econômico, para tanto é fundamental atentar para o fato que a dinâmica econômica das nações depende tanto da demanda - velocidade de adoção e absorção de inovações - como da oferta - produção e implementação de inovações.

- A segunda função da inovação é satisfazer necessidades nacionais e locais, que devem estar articuladas como demandas. Os SI têm legitimidade limitada se a inovação que oferecem não responde às necessidades de suas próprias populações, ou seja, se não estiverem orientadas para a demanda local.

- A terceira função da inovação diz respeito a enfrentar desafios globais, porquanto não basta produzir tecnologias cada vez mais sofisticadas se essas não atingem toda a população necessitada e/ou não serão suficientes para enfrentar desafios existentes. Isso significa que a demanda deve ser articulada e conectada com a oferta, e potenciais compradores e usuários devem ser capazes de entender e usar inovações que atendam aos desafios existentes (EDLER, 2016).

Se faz necessário atentar para a necessidade de identificar o impacto social e econômico das políticas de inovação, assim como os mecanismos que se apresentam como a melhor alternativa para o desenvolvimento de políticas em uma sociedade que, cada vez mais, é baseada no conhecimento e na aprendizagem (LUNDVAL; BORRÁS,1997). Nessa perspectiva, a próxima seção se dedica a apresentar e analisar as políticas públicas voltadas à inovação vigentes no Brasil.

\section{Políticas Públicas em CT\&I Brasileiras}

No Brasil, as diretrizes voltadas à promoção e geração de inovação encontram-se amparadas por um conjunto de políticas públicas, majoritariamente do tipo regulatórias, que visam estabelecer diretrizes e caminhos para a atividade inovativa no país.

Um importante marco dessa trajetória é a Lei no 10.973, de 2 de dezembro de 2004,

(c) RDBCl: Rev. Digit. Bibliotecon. Cienc. Inf. $\quad$ Campinas, SP

v.17

1-14

e019019




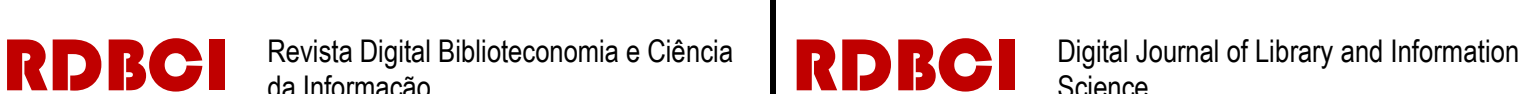

que teve o texto amplamente atualizado pela Lei no 13.243, de 11 de janeiro de 2016, resultando no 'Novo Marco Legal da Inovação' que, amparada por uma emenda constitucional, altera nove legislações determinantes em diferentes segmentos implicados nas atividades de inovação, como descrito no Quadro 1.

Quadro 1. Segmentos Envolvidos na Lei 13.243.

\begin{tabular}{|c|c|}
\hline Segmento implicado & Legislação alterada pela Lei 13.243 \\
\hline Pesquisa científica e tecnológica & $\begin{array}{l}\text { Lei no } 10.973 \text {, de } 2 \text { de dezembro de } 2004 \\
\text { Emenda Constitucional no } 85 \text {, de } 26 \text { de fevereiro de } 2015\end{array}$ \\
\hline Questões imigratórias & Lei no 6.815 , de 19 de agosto de 1980 \\
\hline Compras e contratações públicas & Lei ${ }^{\circ} 8.666$, de 21 de junho de 1993 \\
\hline Instituições de educação e pesquisa & Lei ${ }^{\circ} 8.958$, de 20 de dezembro de 1994 \\
\hline $\begin{array}{l}\text { Importações de bens destinados ao } \\
\text { desenvolvimento científico e tecnológico }\end{array}$ & $\begin{array}{l}\text { Lei } \mathrm{n}^{\circ} 8.010 \text {, de } 29 \text { de março de } 1990 \\
\text { Lei } \mathrm{n}^{\circ} 8.032 \text {, de } 12 \text { de abril de } 1990\end{array}$ \\
\hline $\begin{array}{l}\text { Contratação e desenvolvimento de carreiras de } \\
\text { pessoal potencialmente envolvido com ciência e } \\
\text { tecnologia }\end{array}$ & $\begin{array}{l}\text { Lei } \mathrm{n}^{\circ} 8.745, \text { de } 9 \text { de dezembro de } 1993 \\
\text { Lei } n^{\circ} 12.772 \text {, de } 28 \text { de dezembro de } 2012\end{array}$ \\
\hline
\end{tabular}

Fonte: Elaborado pela autora, 2019.

Além das políticas regulatórias, no que tange a políticas de inovação o contexto brasileiro é também contemplado com a 'Estratégia Nacional de Ciência, Tecnologia e Inovação' (ENCTI), com vigência no período compreendido entre os anos de 2016 e 2019; uma Projeto de Lei (PL) do Senado Federal para a criação de um Plano Nacional de Ciência, Tecnologia e Inovação, e o Programa 2021 'Ciência, Tecnologia e Inovação' que integra o Plano Plurianual 2016-2019.

\section{Procedimentos Metodológicos}

Em pesquisa desenvolvida por Silva (2018), foram analisadas as políticas públicas voltadas à inovação no Brasil, que correspondem às políticas públicas em Ciência, Tecnologia e Inovação $(\mathrm{CT} \& \mathrm{I})$. A análise enfocou questões relacionadas à produção e o compartilhamento de conhecimento no âmbito do SNI brasileiro. Na referida pesquisa aplicou-se do método 'Análise de Conteúdo' com categorias de análise definidas à posteriori. O presente artigo apresenta um recorte da pesquisa supracitada e, dentre as categorias analisadas na pesquisa de Silva (2108), duas são selecionadas devido ao fato de evidenciar aspectos relacionados à atuação integrada entre os agentes do SNI, que indicam processos de inovação aberta, são elas:

1 - Cultura de inovação enfocando o conhecimento: Cultura organizacional voltada à produção de conhecimento entre agentes do SI; Cultura organizacional voltada ao compartilhamento de conhecimento entre agentes do SI. 


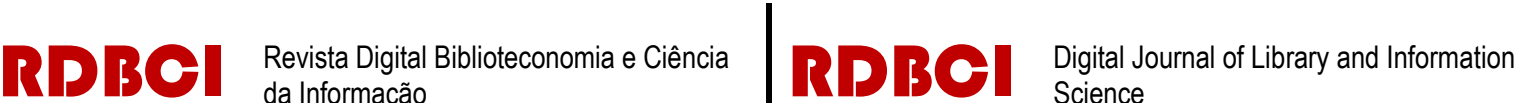

2 - Ação integrada de agentes de SI: Manifestação de ações integradas envolvendo múltiplos agentes com foco na inovação; Manifestação de ações integradas envolvendo múltiplos agentes do SI no que tange à produção e compartilhamento de conhecimento.

A análise das categorias selecionadas possibilitam perceber o enfoque dado nas políticas públicas em CT\&I ao incentivo [ou a ausência deste] à inovação aberta no contexto brasileiro. Assim, um recorte dos resultados da referido pesquisa são objeto da seção seguinte.

\section{Apresentação e Análise de Resultados}

Em relação à categoria 'Cultura de inovação enfocando o conhecimento' as políticas públicas de abrangência nacional revelaram a presença de elementos promotores de uma cultura voltada à produção de conhecimento para a inovação e ao compartilhamento de informação e conhecimento entre os agentes do SNI. Tais elementos se comprovam nas políticas que recomendam que sejam estabelecidos incentivos para a promoção de atividades científica e tecnológicas vinculadas ao SNI; promoção de cooperação entre os agentes do SNI; estímulo às atividades inovativas; e consolidação de ambientes promotores da inovação tais como parques e polos tecnológicos e incubadoras de empresas.

As políticas preveem o compartilhamento de infraestrutura como instalações, laboratórios, equipamentos, instrumentos e materiais sem o prejuízo das atividades principais dos agentes envolvidos. Essa prática pode contribuir com a cultura sistêmica de inovação focada no compartilhamento entre os agentes do SNI, entretanto dois fatores devem ser considerados: 1) o simples compartilhamento de infraestrutura não garante a atuação integrada, mas simplesmente o uso compartilhado de recursos; o ideal é que processos e resultados de produção de conhecimento possam ser compartilhados, pois gerarão novos conhecimentos para todos os agentes envolvidos, e 2) a produção de conhecimento no âmbito de agentes públicos tais como universidades e institutos de pesquisa não pode ficar subjugada aos interesses de organizações empresarias, é preciso que a produção de conhecimento científico esteja atenta às demandas do contexto de aplicação, mas não pode limitar-se a tais necessidades, pois comprometeria o desenvolvimento do conhecimento de fronteira e a autonomia dos pesquisadores.

Foi evidenciado que as políticas públicas de abrangência nacional foram elaboradas com o intuito de promover a construção uma de cultura de inovação enfocando o conhecimento. Entretanto é possível inferir que esta cultura buscada ainda não está consolidada. A assertiva é evidenciada na Estratégia Nacional Ciência, Tecnologia e Inovação (ENCTI) que declara que "[...] Maneiras mais eficazes de trabalhar a educação científica da população devem ser desenvolvidas a fim de estabelecer expectativas elevadas para todos, motivando os alunos à

v.17

$1-14$

e019019
2019 


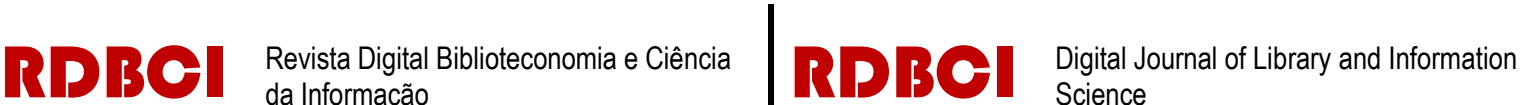

experimentação e atraindo mais cidadãos para as carreiras de CT\&I" (MINISTÉRIO, 2016, p.68).

No âmbito da categoria 'Ações integradas entre agentes do SI' se evidencia a presença tanto de manifestações de ações conjuntas envolvendo os agentes do SI de modo geral, quanto ações conjuntas específicas para a produção e compartilhamento de conhecimento. nessa perspectiva, se espera que agentes públicos de coordenação estimulem e apoiem alianças estratégicas e projetos de cooperação para a "[...] a geração de produtos, processos e serviços inovadores e a transferência e a difusão de tecnologia" (BRASIL, 2016, p.3). Para tanto programas tais como o Plano Brasil Maior, o Plano Inova Empresa, o Sistema Brasileiro de Tecnologia (SIBRATEC), e a Empresa Brasileira de Pesquisa e Inovação Industrial (EMBRAPII), são citados na ENCTI como elementos aceleradores do desenvolvimento científico e tecnológico do país.

A ENCTI declara que crescem as medidas para o [...] aprimoramento dos recursos humanos e a melhoria das condições estruturais para inovação, priorizando uma maior interação entre pesquisa pública e indústria (MINISTÉRIO..., 2016, p.51), levando em consideração especificidades locais, setoriais, regionais, nacional e global. $\mathrm{O}$ compartilhamento de recursos humanos entre universidades e empresas é visto como fundamental para a promoção da inovação, e por conseguinte são incentivadas ações que possibilitem o acesso de empresas às competências e estruturas da universidades. Além disso, a Estratégia propõe que sejam realizados investimentos na modernização, recuperação e fortalecimento da infraestrutura de pesquisa no país.

No entanto, ainda que a ENCTI destaque os programas voltados à inovação e aprimoramento das relações entre universidade e empresa, cabe destacar que a partir de 2016, mudanças na plataforma do governo federal influenciaram o desenvolvimento das iniciativas citadas (Plano Brasil Maior, Plano Inova Empresa, SIBRATEC, EMBRAPII), tendo algumas sido reestruturadas e outras suprimidas. Defende-se nesta pesquisa a importância do desenvolvimento de estratégias que minimizem a vulnerabilidade de políticas, programas e planos voltados ao desenvolvimento do país, porquanto é preciso que sejam praticadas políticas de Estado e não políticas de governo dependentes de questões político-partidárias.

Em síntese, as políticas públicas de CT\&I em contexto brasileiro enfocam a atuação integrada entre os agentes do SNI e também a atuação integrada no que tange à produção e compartilhamento de conhecimento. Se confirma a presença do tema em praticamente todas as políticas públicas de CT\&I analisadas, especialmente naquelas que traduzem os princípios e diretrizes que devem ser buscados pelo país. No entanto, se nota uma deficiência no que tange a planos que deveriam descrever projetos e ações a serem executados para o alcance dos objetivos traçados nas políticas norteadoras, contemplando de maneira clara e objetiva os

\begin{tabular}{c|c|c|c|c|c}
\hline (c) RDBCl: Rev. Digit. Bibliotecon. Cienc. Inf. & Campinas, SP & v.17 & $1-14$ & e019019 & 2019 \\
\hline
\end{tabular}




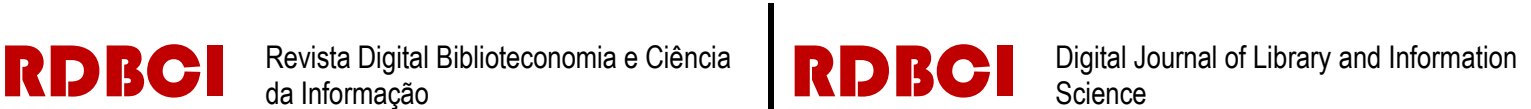

projetos e respectivas ações necessárias para o alcance dos objetivos traçados no âmbito das políticas norteadoras.

É importante ressaltar que se observa uma grande quantidade de políticas regulatórias que dispõem sobre incentivos à inovação, regimes de contratação e permanência de pessoal envolvido com CT\&I e meios de obtenção de recursos e infraestrutura. Entretanto, os enfoques das referidas políticas regulatórias são resgatados na ENCTI, embora não de maneira direta. No que tange à ENCTI, esta mantém uma estrutura bastante abrangente, abordando os enfoques do Sistema Nacional de Ciência, Tecnologia e Inovação (SNCTI), os desafios nacionais, os pilares fundamentais do SNI e os temas estratégicos. Para estes (temas estratégicos) são apresentadas estratégias associadas tais como a elaboração de planos específicos no contexto dos temas, criação e/ou fortalecimentos de parcerias, fomento à PD\&I em áreas específicas, entre outras. Entretanto, o conjunto de políticas públicas de CT\&I brasileiro carece de um plano nacional em CT\&I.

A participação da sociedade civil em processos inovativos, de maneira independente de vínculos organizacionais praticamente não é contemplada nas políticas públicas analisadas.

\section{Considerações Finais}

A inovação ocupa um importante papel no desenvolvimento das nações e deve ser objeto de atenção tanto do setor produtivo, quanto do acadêmico e também do segmento governamental. Este último tem entre suas responsabilidades, ocupar-se da elaboração de políticas públicas capazes de viabilizar a atuação integrada dos diferentes agentes do SNI e, por consequência, promover o desenvolvimento.

A análise das políticas públicas brasileiras evidenciou o incentivo à cooperação entre os agentes do SNI, prevendo o compartilhamento de infraestrutura como instalações, laboratórios, equipamentos, instrumentos e materiais sem o prejuízo das atividades principais dos agentes envolvidos. Também se observa a priorização da interação universidade e indústria, inclusive por meio do compartilhamento de recursos humanos entre universidades e empresas. Entretanto, as políticas observadas não contemplam o incentivo da participação de pessoas não vinculadas às organizações integrantes do SNI, aspecto que caracteriza-se como uma tendência e, portanto, poderia ser melhor explorado pelas políticas públicas.

Futuros estudos poderão abordar investigações acerca da elaboração e implantação de políticas públicas que contemplem e facilitem o envolvimento de pessoas não vinculadas a agentes de sistemas de inovação, tanto no que tange a desenvolvimentos inovativos quanto em processos de financiamento coletivo. Acredita-se políticas públicas em CT\&I ainda mais

\begin{tabular}{l|l|l|l|l|l|}
\hline C RDBCl: Rev. Digit. Bibliotecon. Cienc. Inf. & Campinas, SP & v.17 & $1-14$ & e019019 & 2019 \\
\hline
\end{tabular}


abrangentes poderão fomentar o fortalecimento de ecossistemas de inovação promotores de atuação em rede e cocriação.

\section{Referências}

BRASIL. Ministério da Ciência, Tecnologia e Inovação. Estratégia nacional de ciência, tecnologia e inovação 2016-2019. Brasília: Ministério da Ciência, Tecnologia e Inovação, 2016.

BRASIL. Presidência da República. Casa Civil. Lei nº13.243, de 11 de janeiro de 2016. Dispõe sobre estímulos ao desenvolvimento científico, à pesquisa, à capacitação científica e tecnológica e à inovação e altera a Lei no 10.973, de 2 de dezembro de 2004, a Lei no 6.815, de 19 de agosto de 1980, a Lei no 8.666, de 21 de junho de 1993, a Lei no 12.462, de 4 de agosto de 2011, a Lei no 8.745, de 9 de dezembro de 1993, a Lei no 8.958, de 20 de dezembro de 1994, a Lei no 8.010, de 29 de março de 1990, a Lei no 8.032, de 12 de abril de 1990, e a Lei no 12.772, de 28 de dezembro de 2012, nos termos da Emenda Constitucional no 85, de 26 de fevereiro de 2015. Brasília: 2016. Disponível em: <http://www.planalto.gov.br/ccivil_03/_ato2015-2018/2016/lei/113243.htm>. Acesso em 12 jun. 2017.

CHESBROUGH, H. W. Open innovation: The new imperative for creating and profiting from technology. Boston: Harvard Business Scholl Press, 2003.

CORNELL University; INSEAD; WIPO. The global innovation index 2016: winning with global innovation. Geneva: WIPO, 2016.

<http://www.globalinnovationindex.org/gii >. Acesso em: 10 jun. 2016.

EDLER, J. Local Needs, Global Challenges: The Meaning of Demand-Side Policies for Innovation and Development. In: CORNELL University; INSEAD; WIPO. The Global Innovation Index 2016: Winning with Global Innovation. Geneva: WIPO, 2016. p.97-102.

EDQUIST, C. Systems of innovation approaches: Their emergence and characteristics. In: EDQUIST, C. (Ed.). Systems of innovation: technologies, institutions and organizations. London; Washington: Pinter, 1997.

ETZKOWITZ, H.; LEYDESDORFF, L. The dynamics of innovation: from National Systems and "Mode 2"' to a Triple Helix of university-industry-government relations. Research Policy, v. 29, n.2, p.109-123, Feb. 2000.

FERNANDES. A. S. A. Políticas públicas: definição, evolução e o caso brasileiro na política social. In: DANTAS, H.; MATINS JR., J. P. (Org.). Introdução à política brasileira. São

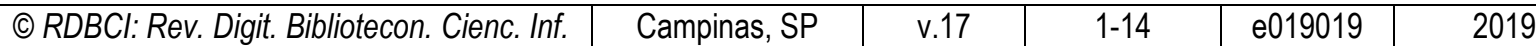


Paulo: Paulus, 2007.

FREEMAN, C.; SOETE, L. A economia da inovação industrial. Campinas (SP): Editora UNICAMP, 2008.

FIGUEIREDO, P. N. G. Gestão da Inovação: conceitos, métricas e experiências de empresas no Brasil. Rio de Janeiro: LTC, 2012.

GODIN, B. National Innovation System: The system approach in historical perspective.

Science, Technology \&HumanValues, v.34, n.4, p.476-501, Jul. 2009.

JOHNSON, B. Institutional Learning. In: LUNDVALL, B. A. (Ed.). National systems of innovation: Towards a theory of innovation and interactive learning. London: Pinter Publishers, 1992.

LIST, F. The national system of political economy. London, Longman, 1904.

LUNDVALL, B. A. et.al. National systems of production, innovation and competence building. Research Policy, v.31, n.2, p.213-231, Feb. 2002.

LUNDVALL, B. A.; BORRÁS, S. The globalising learning economy: Implications for innovation policy. Aalborg; Copenhagen, 1997. Disponível em:

http://www.globelicsacademy.org/2011_pdf/Lundvall\%20Borras\%201997.pdf. Acesso em: 10 jan. 2014.

MOORE, J. E. Business ecosystems and the view from the firm. The Antitrust Bulletin, v. 51, n. 1, 2006.

ORGANISATION FOR ECONOMIC CO-OPERATION AND DEVELOPMENT. Open innovation in global networks. Report. New York: OECD, 2008.

ORGANISATION FOR ECONOMIC CO-OPERATION AND DEVELOPMENT. Oslo manual: the measurement of scientific and technological activities. Proposed guidelines for collecting and interpreting technological innovation data. [s.1.]: European Comission, Eurostat, 2005.

ORGANISATION FOR ECONOMIC CO-OPERATION AND DEVELOPMENT. National Innovation Systems. Paris: OECD, 1997. 
SCHUMPETER, J. A. Teoria do desenvolvimento econômico. São Paulo: Abril Cultural, 1982.

SILVA, E. da. O conhecimento científico no contexto de sistemas nacionais de inovação: análise de políticas públicas e indicadores de inovação. 281f. Tese (Doutorado) - Programa de Pós-Graduação em Ciência da Informação, Faculdade de Filosofia e Ciências, Universidade Estadual Paulista (Unesp), Marília, 2018.

STAL, E.; NOHARA, J. J.; CHAGAS JÚNIOR, M.F. Os conceitos da inovação aberta e o desempenho de empresas brasileiras inovadoras. Revista de Administração e Inovação, v.11, n.2, p.295-320, abr./jun. 2014.

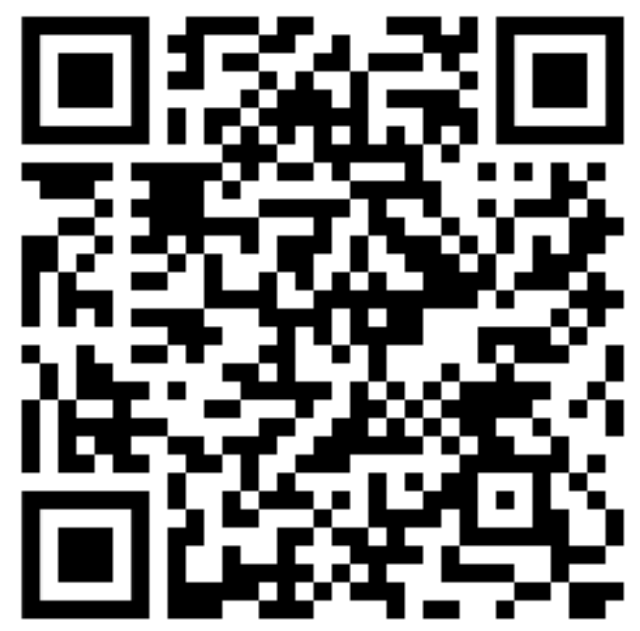

